NTERnational Electronic Journal of Algebra

VOLUME 31 (2022) 1-12

DOI: $10.24330 /$ ieja.1058380

\title{
ON A PROPERTY OF THE IDEALS OF THE POLYNOMIAL RING $R[x]$
}

\author{
Amr Ali Abdulkader Al-Maktry \\ Received: 3 April 2020; Revised: 2 February 2021; Accepted: 23 April 2021 \\ Communicated by Burcu Üngör

\begin{abstract}
Let $R$ be a commutative ring with unity $1 \neq 0$. In this paper we introduce the definition of the first derivative property on the ideals of the polynomial ring $R[x]$. In particular, when $R$ is a finite local ring with principal maximal ideal $\mathfrak{m} \neq\{0\}$ of index of nilpotency $e$, where $1<e \leq|R / \mathfrak{m}|+1$, we show that the null ideal consisting of polynomials inducing the zero function on $R$ satisfies this property. As an application, when $R$ is a finite local ring with null ideal satisfying this property, we prove that the stabilizer group of $R$ in the group of polynomial permutations on the ring $R[x] /\left(x^{2}\right)$, is isomorphic to a certain factor group of the null ideal.
\end{abstract}

Mathematics Subject Classification (2020): 13F20, 06B10, 13J15, 11T06, 05A05, 13B25, 20B35

Keywords: Commutative rings, polynomial ring, null ideal, null polynomial, Henselian ring, finite local ring, dual numbers, polynomial permutation, permutation polynomial, finite permutation group

\section{Introduction}

Let $R$ be a commutative ring with unity $1 \neq 0$, and $R[x]$ be the polynomial ring over $R$ of one indeterminate $x$. In addition to the usual operations on polynomials, $R[x]$ has a further operation, which appears in a normal way, namely the formal derivative of polynomials. Nöbauer used this operation to define the derivative of ideals with a certain property [4].

Another well known feature of $R[x]$ is that every polynomial $f(x)=\sum_{j=0}^{k} a_{j} x^{j} \in$ $R[x]$ induces a function $F: R \longrightarrow R$, where $F(r)=\sum_{j=0}^{k} a_{j} r^{j}$ for all $r \in R$. In this case $F$ is called a polynomial function on $R$. The set of all polynomial functions on $R$ is a monoid via composition of functions. Moreover, when the function $F$ is a bijection we say $F$ is a polynomial permutation while $f$ is a permutation polynomial. Obviously, the set of all polynomial permutations is a group, which 
we denote by $\mathcal{P}(R)$. Further, $\mathcal{P}(R)$ forms the group of units of the monoid of polynomial functions.

If a polynomial $g \in R[x]$ induces the constant zero function over $R$, that is $g(r)=0$ for each $r \in R$, then $g$ is called a null polynomial over (on) $R$. The set of all null polynomials on $R$ is an ideal of $R[x]$, which we denote by $N_{R}$ and we call it the null ideal (on $R$ ). The null ideal $N_{R}$ supplies the ring of polynomials $R[x]$ with an equivalence relation in which two polynomials $g, h \in R[x]$ are equivalent whenever $g-h \in N_{R}$. In other words, two polynomials are related in this relation if and only if they induce the same function on $R$. Moreover, every equivalence class corresponds to one polynomial function on $R$ and vice versa.

This paper considers a property of the ideals of the ring $R[x]$ and its application to the group of polynomial permutations on finite rings. In particular, for a finite local ring $R$ with null ideal having this property, we prove some facts about the permutation polynomials on the ring $R[x] /\left(x^{2}\right)$.

The property defined in the paper depends on the formal derivative of polynomials, however it is completely different from the one considered in [4].

Throughout this paper for a local ring $R$, let $\mathfrak{m}$ denote its maximal ideal and let $N(\mathfrak{m})$ be the set of all polynomials over $R$ which vanish on the ideal $\mathfrak{m}$. Evidently, $N(\mathfrak{m})$ is an ideal in the polynomial ring $R[x]$ containing $N_{R}$. For $f \in R[x]$ with $f(x)=\sum_{i=0}^{n} a_{i} x^{i}$, let $f^{\prime}$ denote its formal derivative; i.e., $f^{\prime}(x)=\sum_{i=1}^{n} i a_{i} x^{i-1}$.

\section{The first derivative property and the null ideal}

We begin this section with the definition of our property. Then we prove some supplementary results. Later, we show that the null ideal $N_{R}$ has this property for a wide class of finite local rings with principal maximal ideals.

Definition 2.1. Let $R$ be a commutative ring. An ideal $I$ of $R[x]$ satisfies the first derivative property if $g, h \in I$ implies that $g^{\prime} h^{\prime} \in I$.

For shortness we use the abbreviation FDP for the first derivative property.

Proposition 2.2. Let $I, J$ be ideals of $R[x]$. Then:

(1) $I^{2}$ satisfies FDP;

(2) if $I$ and $J$ satisfy FDP, then $I J$ satisfies FDP.

Proof. We prove (2) and leave (1) to the reader. Let $f, g \in I J$. Then there exist polynomials $f_{1}, \ldots, f_{n} ; g_{1}, \ldots, g_{m} \in I$ and $h_{1}, \ldots, h_{n} ; k_{1}, \ldots, k_{m} \in J$ such that $f=\sum_{i=1}^{n} f_{i} h_{i}$ and $g=\sum_{j=1}^{m} g_{j} k_{j}$. So $f^{\prime}=\sum_{i=1}^{n} f_{i}^{\prime} h_{i}+\sum_{i=1}^{n} f_{i} h_{i}^{\prime}$ and $g^{\prime}=\sum_{j=1}^{m} g_{j}^{\prime} k_{j}+$ 
$\sum_{j=1}^{m} g_{j} k_{j}^{\prime}$. Obviously, $\sum_{i=1}^{n} f_{i} h_{i}^{\prime}, \sum_{j=1}^{m} g_{j} k_{j}^{\prime} \in I$ and $\sum_{i=1}^{n} f_{i}^{\prime} h_{i}, \sum_{j=1}^{m} g_{j}^{\prime} k_{j} \in J$. On the other hand, by Definition 2.1, we have $f_{i}^{\prime} g_{j}^{\prime} \in I$ for every $1 \leq i \leq n ; 1 \leq j \leq m$. Hence $\left(\sum_{i=1}^{n} f_{i}^{\prime} h_{i}\right)\left(\sum_{j=1}^{m} g_{j}^{\prime} k_{j}\right)=\sum_{i, j} f_{i}^{\prime} g_{j}^{\prime} h_{i} k_{j} \in I J$. Similarly, $\left(\sum_{i=1}^{n} f_{i} h_{i}^{\prime}\right)\left(\sum_{j=1}^{m} g_{j} k_{j}^{\prime}\right) \in I J$. Therefore

$$
f^{\prime} g^{\prime}=\left(\sum_{i=1}^{n} f_{i}^{\prime} h_{i}+\sum_{i=1}^{n} f_{i} h_{i}^{\prime}\right)\left(\sum_{j=1}^{m} g_{j}^{\prime} k_{j}+\sum_{j=1}^{m} g_{j} k_{j}^{\prime}\right) \in I J
$$

The following result gives a criterion for FDP for finitely generated ideals over $R[x]$.

Proposition 2.3. Let $I$ be an ideal of $R[x]$ and suppose that $I=\left(f_{1}, \ldots, f_{n}\right)$ for some $f_{1}, \ldots, f_{n} \in R[x]$. Then I satisfies FDP if and only if $f_{i}^{\prime} f_{j}^{\prime} \in I$ for all $i, j \in\{1, \ldots, n\}$.

Proof. $(\Rightarrow)$ Obvious.

$(\Leftarrow)$ Suppose that $f_{i}^{\prime} f_{j}^{\prime} \in I$ for any two generators $f_{i}, f_{j} \in I$. Let $g, h \in I$. Then there exist $g_{1}, \ldots, g_{n} ; h_{1}, \ldots, h_{n} \in R[x]$ such that $g(x)=\sum_{i=1}^{n} g_{i} f_{i}$ and $h(x)=$ $\sum_{i=1}^{n} h_{i} f_{i}$. We have $g^{\prime}=\sum_{i=1}^{n} g_{i}^{\prime} f_{i}+\sum_{i=1}^{n} g_{i} f_{i}^{\prime}$ and $h^{\prime}=\sum_{i=1}^{n} h_{i}^{\prime} f_{i}+\sum_{i=1}^{n} h_{i} f_{i}^{\prime}$. So

$$
g^{\prime} h^{\prime}=\left(\sum_{i=1}^{n} g_{i}^{\prime} f_{i}\right) h^{\prime}+\left(\sum_{i=1}^{n} g_{i} f_{i}^{\prime}\right)\left(\sum_{i=1}^{n} h_{i}^{\prime} f_{i}\right)+\left(\sum_{i, j=1}^{n} g_{i} h_{j} f_{i}^{\prime} f_{j}^{\prime}\right) .
$$

Clearly, $g^{\prime} h^{\prime} \in I$, and hence $I$ satisfies FDP.

Remark 2.4. Let $R$ be a local ring with maximal ideal $\mathfrak{m}$ and residue field $\mathbb{F}_{q}$, and let $\lambda(x)=\prod_{i=1}^{q}\left(x-c_{i}\right)$, where $\left\{c_{1}, \ldots, c_{q}\right\}$ is any complete systems of residue modulo $\mathfrak{m}$. It is obvious that $c_{i}-c_{j}$ is a unit in $R$ whenever $i \neq j$; hence for every $r \in R$ such that $r \equiv c_{i} \bmod \mathfrak{m}, r-c_{j}$ is a unit. Then the following lemma follows.

Lemma 2.5. Let $r \in R$. Then $\lambda^{\prime}(r)$ is a unit in $R$.

Remark 2.6. It is a celebrated fact that every finite local commutative ring is a Henselian ring, i.e., a local ring in which Hensel's lemma holds, (see for example, [2, Theorem. XIII.4]). This allows us to use some facts about the ideals $\mathfrak{m}, N_{R}$, when $R$ is a Henselian ring, from [5] to improve our related ideas on finite local rings.

Lemma 2.7. [5, Corollary 2.11] Let $R$ be a Henselian ring. Then $\lambda(R)=\mathfrak{m}$.

Lemma 2.8. [5, Theorem 4.2] Let $R$ be a Henselian ring and $\lambda(x)$ as in Remark 2.4. If $N(\mathfrak{m})=\left(F_{1}(x), \ldots, F_{n}(x)\right)$, then $N_{R}=\left(F_{1}(\lambda(x)), \ldots, F_{n}(\lambda(x))\right)$. 
Recall from the introduction the definitions of the ideals $N_{R}, N(\mathfrak{m})$. The following result shows that, for a Henselian ring $R$ and a finitely generated ideal $N(\mathfrak{m})$, either both $N(\mathfrak{m})$ and $N_{R}$ satisfy FDP or neither satisfies FDP.

Theorem 2.9. Let $R$ be a Henselian ring and $\lambda(x)$ as in Remark 2.4. If $N(\mathfrak{m})=$ $\left(F_{1}(x), \ldots, F_{n}(x)\right)$, then $N_{R}$ satisfies FDP if and only if $N(\mathfrak{m})$ satisfies FDP.

Proof. By Lemma 2.8, $N_{R}=\left(F_{1}(\lambda(x)), \ldots, F_{n}(\lambda(x))\right)$.

$(\Leftarrow)$ Suppose that $N(\mathfrak{m})$ satisfies FDP. Then for every $i, j \in\{1, \ldots, n\}$ there exists $h_{i, j} \in N(\mathfrak{m})$ such that $F_{i}^{\prime} F_{j}^{\prime}=h_{i, j}$. Hence $F_{i}^{\prime}(\lambda(x)) F_{j}^{\prime}(\lambda(x))=h_{i, j}(\lambda(x)) \in$ $N_{R}$ since $\lambda(R)=\mathfrak{m}$ by Lemma 2.7. Now

$$
\left(F_{i}(\lambda(x))\right)^{\prime}\left(F_{j}(\lambda(x))\right)^{\prime}=\left(\lambda^{\prime}(x)\right)^{2} F_{i}^{\prime}(\lambda(x)) F_{j}^{\prime}(\lambda(x))=\left(\lambda^{\prime}(x)\right)^{2} h_{i, j}(\lambda(x)) \in N_{R} .
$$

Thus $N_{R}$ satisfies FDP by Proposition 2.3.

$(\Rightarrow)$ Suppose that $N_{R}$ satisfies FDP. Then for every $i, j \in\{1, \ldots, n\}$ we have

$$
\left(F_{i}(\lambda(x))\right)^{\prime}\left(F_{j}(\lambda(x))\right)^{\prime}=\left(\lambda^{\prime}(x)\right)^{2} F_{i}^{\prime}(\lambda(x)) F_{j}^{\prime}(\lambda(x)) \in N_{R}
$$

Now let $r \in R$ be arbitrary, so $\left(\lambda^{\prime}(r)\right)^{2} F_{i}^{\prime}(\lambda(r)) F_{j}^{\prime}(\lambda(r))=0$ by the definition of $N_{R}$. Hence $F_{i}^{\prime}(\lambda(r)) F_{j}^{\prime}(\lambda(r))=0$ since $\lambda^{\prime}(r)$ is a unit by Lemma 2.5, whence $F_{i}^{\prime}(\lambda(x)) F_{j}^{\prime}(\lambda(x)) \in N_{R}$. But, $\lambda(R)=\mathfrak{m}$ by Lemma 2.7. Therefore $F_{i}^{\prime} F_{j}^{\prime} \in N(\mathfrak{m})$ for every $i, j \in\{1, \ldots, n\}$. Thus $N(\mathfrak{m})$ satisfies FDP by Proposition 2.3.

Remark 2.10. Notice that we don't require $R$ to be Noetherian. In fact there exists a Henselian ring which is non-Noetherian with a finitely generated ideal $N(\mathfrak{m})$ (see [5, Example 3.2]).

Our aim now is to show that the null ideal $N_{R}$ satisfies FDP for every finite local ring with a nonzero principal maximal ideal of index of nilpotency less than or equal $q+1$, where $q$ is the cardinality of the residue field $\mathbb{F}_{q}$. To do so, we need this lemma.

Lemma 2.11. [5, Theorem 4.4] Let $R$ be a finite local ring with principal maximal ideal $\mathfrak{m}=(m)$ and residue filed $\mathbb{F}_{q}$. Suppose $e$ is the index of nilpotency of $\mathfrak{m}$. If $e \leq q$, then $N(\mathfrak{m})=(x, m)^{e}$; if $e=q+1$, then $N(\mathfrak{m})=(x, m)^{e}+\left(x^{q}-m^{q-1} x\right)$.

Theorem 2.12. Let $R$ be a finite local ring with principal maximal ideal $\mathfrak{m}=(m)$ and residue filed $\mathbb{F}_{q}$. Suppose $e$ is the index of nilpotency of $\mathfrak{m}$. If $1<e \leq q+1$ then $N_{R}$ satisfies FDP, provided $e \geq 4$ when $e=q+1$. 
Proof. In view of Theorem 2.9, we need only to prove that $N(\mathfrak{m})$ satisfies FDP. Now $N(\mathfrak{m})$ is finitely generated since $R$ is finite, so it is enough to show that $g^{\prime} h^{\prime} \in N(\mathfrak{m})$ for every pair of generators $g, h$ of $N(\mathfrak{m})$ by Proposition 2.3.

First assume that $1<e<q+1$. By Lemma $2.11, N(\mathfrak{m})$ is generated by the set $\left\{x^{e}, m x^{e-1}, \ldots, m^{e-1} x\right\}$. Let $g, h$ be any generators of $N(\mathfrak{m})$. Then $g(x)=m^{j} x^{e-j}$ and $h(x)=m^{i} x^{e-i}$ for some $0 \leq i, j \leq e-1$. Therefore

$$
g^{\prime}(x) h^{\prime}(x)=(e-i)(e-j) m^{i+j} x^{2 e-i-j-2} \in N(\mathfrak{m})
$$

since $e \geq 2$, and so $N(\mathfrak{m})$ satisfies FDP.

We now consider the case $e=q+1$. By Lemma 2.11, $N(\mathfrak{m})$ is generated by the following set $\left\{x^{e}, m x^{e-1}, \ldots, m^{e-1} x, x^{q}-m^{q-1} x\right\}$. Since $q \in \mathfrak{m}$ we have $q=r m$ for some $r \in R$. Let $g, h$ any two generators of $N(\mathfrak{m})$. We distinguish three cases.

Case 1. $g(x)=h(x)=x^{q}-m^{q-1} x$. Then

$$
g^{\prime}(x) h^{\prime}(x)=\left(q x^{q-1}-m^{q-1}\right)^{2}=q^{2} x^{2 q-2}-2 q m^{q-1} x^{q-1}+m^{2 q-2},
$$

whence

$$
g^{\prime}(x) h^{\prime}(x)=r^{2} m^{2} x^{2 e-4}-2 r m^{e-1} x^{e-2}+m^{2 e-4} .
$$

Evidently, $r^{2} m^{2} x^{2 e-4}-2 r m^{e-1} x^{e-2} \in N(\mathfrak{m})$ since $e=q+1 \geq 3$. Thus $g^{\prime} h^{\prime} \in N(\mathfrak{m})$ if and only if $m^{2 e-4} \in N(\mathfrak{m})$ if and only if $m^{2 e-4}=0$, provided $e \geq 4$.

Case 2. $g(x)=x^{q}-m^{q-1} x$ and $h(x)=m^{i} x^{e-i}$ for some $0 \leq i \leq e-1$.

Then

$g^{\prime}(x) h^{\prime}(x)=(e-i) m^{i} x^{e-i-1}\left(q x^{q-1}-m^{q-1}\right)=(e-i) m^{i} x^{e-i-1}\left(r m x^{e-2}-m^{e-2}\right)=$ $=(e-i) m^{i+1} x^{e-i-1}\left(r x^{e-2}-m^{e-3}\right) \in N(\mathfrak{m})$ since $m^{i+1} x^{e-i-1} \in N(\mathfrak{m})$ and $e \geq$ $4>3$.

Case 3. $g(x)=m^{j} x^{e-j}$ and $h(x)=m^{i} x^{e-i}$ for some $0 \leq i, j \leq e-1$. Then

$$
g^{\prime}(x) h^{\prime}(x)=(e-i)(e-j) m^{i+j} x^{2 e-i-j-2} \in N(\mathfrak{m})
$$

since $e \geq 4$.

Therefore $N(\mathfrak{m})$ satisfies FDP.

Remark 2.13. (1) If $e=1$, then $R=\mathbb{F}_{q}$. In this case $N_{\mathbb{F}_{q}}=\left(x^{q}-x\right) \mathbb{F}_{q}[x]$. But, $N_{\mathbb{F}_{q}}$ does not satisfy FDP. Because, if we take $g(x)=x^{q}-x$, then $\left(g^{\prime}(x)\right)^{2}=\left(q x^{q-1}-1\right)^{2}=1 \notin N_{\mathbb{F}_{q}}$. 
(2) Consider $g(x)=\left(x^{2}-x\right)^{2}-2\left(x^{2}-x\right) \in \mathbb{Z}_{8}[x]$, by Fermat's Theorem, one can show easily that $g(a) \equiv 0(\bmod 8)$ for every $a \in \mathbb{Z}_{8}$, that is, $g \in N_{\mathbb{Z}_{8}}$. However, $N_{\mathbb{Z}_{8}}$ does not satisfy FDP since $\left(g^{\prime}\right)^{2} \notin N_{\mathbb{Z}_{8}}$. Indeed, $\left(g^{\prime}(1)\right)^{2}=4 \not \equiv 0(\bmod 8)$. Note that $e=q+1=3<4$.

Corollary 2.14. Let $n$ be a positive integer and $p$ a prime number.

(1) If $p>2$, then $N_{\mathbb{Z}_{p^{n}}}$ satisfies FDP for every $1<n \leq p+1$.

(2) If $p=2$, then $N_{\mathbb{Z}_{4}}$ satisfies FDP.

Although we defined null ideals for finite rings, the definition is still true for infinite rings. We consider this fact in the following example.

Example 2.15. Let $R$ be a boolean ring (not necessary finite). By definition, $f=x^{2}-x \in N_{R}$. But, $\left(f^{\prime}\right)^{2}=(-1)^{2}=1 \notin N_{R}$.

Right now we have achieved our first main goal, that is, showing the existence of a wide class of finite local rings with null ideals having FDP. In the next section we employ FDP to infer some facts about a group of polynomial permutations over the ring $R[x] /\left(x^{2}\right)$.

\section{Applications to polynomial permutations of the ring $R[x] /\left(x^{2}\right)$}

In this section, for a finite local commutative ring $R$ with the null ideal $N_{R}$ satisfying the first derivative property, we prove some facts about some kind of permutation polynomials on the ring $R[x] /\left(x^{2}\right)$.

Throughout this section all rings are finite.

Recall that $R[x] /\left(x^{2}\right)$ is isomorphic to the ring $R[\alpha]=\{a+b \alpha: a, b \in R\}$, where $\alpha \notin R$ and $\alpha^{2}=0$. Here are some easily verifiable facts about polynomials over $R[\alpha]$.

Fact 3.1. Let $h \in R[x]$. Then $h(a+b \alpha)=h(a)+b h^{\prime}(a) \alpha$ for each $a, b \in R$.

Fact 3.2. Let $g \in R[\alpha][x]$. Then $g=g_{1}+g_{2} \alpha$ for some $g_{1}, g_{2} \in R[x]$.

Recall from the introduction that $\mathcal{P}(R[\alpha])$ denotes the group of polynomial permutations on the ring $R[\alpha]$.

Definition 3.3. Let $S t_{\alpha}(R)=\{F \in \mathcal{P}(R[\alpha]): F(r)=r$ for each $r \in R\}$.

Obviously, $S t_{\alpha}(R)$ is a nonempty finite subset of $\mathcal{P}(R[\alpha])$. Further, it is closed under the composition of functions. Therefore $S t_{\alpha}(R)$ is a subgroup of $\mathcal{P}(R[\alpha])$. The group $S t_{\alpha}(R)$ by definition stabilizes every element of $R$; for this we call it 
the stabilizer group of $R$ in the group of polynomial permutations of $R[\alpha]$ or more shortly the stabilizer group.

Lemma 3.4. Let $A$ be a ring and $g, h \in A[x]$. If $g$ and $h$ induce the same function over $A$, then there exists $f \in N_{A}$ such that $g=h+f$.

Proof. Take $f=g-h$. Then $f \in N_{A}$.

We need some facts from [1]. However, we prove these facts as the proofs do not depend on extra materials.

Lemma 3.5. [1, Lemma 3.4] Let $h \in N_{R}$. Then h $\alpha$ induces the zero function over $R[\alpha]$.

Proof. By Fact 3.1, $h(a+b \alpha) \alpha=\left(h(a)+b h^{\prime}(a) \alpha\right) \alpha=h(a) \alpha+0=0 \alpha=0$ for all $a, b \in R$.

Proposition 3.6. [1, Proposition 4.6] Let $R$ be a ring. Then

$$
S t_{\alpha}(R)=\left\{F \in \mathcal{P}(R[\alpha]): F \text { is induced by } x+f(x) \text { for some } f \in N_{R}\right\} .
$$

Proof. It is obvious that

$$
S t_{\alpha}(R) \supseteq\left\{F \in \mathcal{P}(R[\alpha]): F \text { is induced by } x+f(x) \text { for some } f \in N_{R}\right\} \text {. }
$$

Now let $F \in \mathcal{P}(R[\alpha])$ such that $F(r)=r$ for each $r \in R$. Since $F$ is a polynomial permutation over $R[\alpha], F$ is induced by a polynomial $g \in R[\alpha][x]$. By Fact 3.2, $g=g_{0}+g_{1} \alpha$, where $g_{0}, g_{1} \in R[x]$. Now $r=F(r)=g(r)=g_{0}(r)+g_{1}(r) \alpha$ for each $r \in R$. Then $g_{1}(r) \alpha=0$, and so $g_{1}(r)=0$ for each $r \in R$, i.e., $g_{1} \in N_{R}$. Hence, $g_{1} \alpha$ is a null polynomial over $R[\alpha]$ by Lemma 3.5. Thus $g_{0}$ and $g_{0}+g_{1} \alpha$ both induce $F$ on $R[\alpha]$, i.e., $F$ is induced by $g_{0}$. Further, $g_{0} \equiv x \bmod N_{R}$, i.e., $g_{0}$ induces the identity on $R$, and therefore $g_{0}(x)=x+h(x)$ for some $h \in N_{R}$ by Lemma 3.4. This shows the other inclusion.

Lemma 3.7. Let $F \in S t_{\alpha}(R)$. Suppose that $x+f(x)$ induces $F$, where $f \in N_{R}$. Then the following statements are equivalent

(1) $\left(f^{\prime}\right)^{2} \in N_{R}$;

(2) $x-f(x)$ induces $F^{-1}$;

(3) $F^{k}=\underbrace{F \circ F \circ \cdots \circ F}_{k \text { times }}$ is induced by $x+k f(x)$ for every $k \in \mathbb{N}$. 
Proof. (1) $\Rightarrow(2)$ Let $G$ be the function induced by $x-f(x)$. Then for every $r, s \in R$ we have

$$
\begin{aligned}
G \circ F(r+s \alpha) & =G(r+s \alpha+f(r+s \alpha)) \\
& =G\left(r+s \alpha+f(r)+s f^{\prime}(r) \alpha\right) \quad(\text { by Fact 3.1) } \\
& =G\left(r+s \alpha+s f^{\prime}(r) \alpha\right) \quad(\text { since } f \text { is null) } \\
& =\left(r+s \alpha+s f^{\prime}(r) \alpha\right)-f\left(r+\left(s+s f^{\prime}(r)\right) \alpha\right) \\
& =\left(r+s \alpha+s f^{\prime}(r) \alpha\right)-\left(f(r)+\left(s+s f^{\prime}(r)\right) f^{\prime}(r) \alpha\right) \quad \text { by Fact 3.1) } \\
& =r+s \alpha+s f^{\prime}(r) \alpha-s f^{\prime}(r) \alpha \quad\left(\text { since }\left(f^{\prime}\right)^{2} \in N_{R}\right) \\
& =r+s \alpha .
\end{aligned}
$$

Thus $F^{-1}=G$, whence $x-f(x)$ induces $F^{-1}$.

$(2) \Rightarrow(1)$ Let $x-f(x)$ induces $F^{-1}$. Then one can use the previous calculations to get that for every $r, s \in R, r+s \alpha=F^{-1} \circ F(r+s \alpha)=r+s \alpha-s\left(f^{\prime}(r)\right)^{2} \alpha$.

Hence $s\left(f^{\prime}(r)\right)^{2} \alpha=0$, whence $\left(f^{\prime}(r)\right)^{2} s=0$ for every $r, s \in R$. So if $s=1$, we have $\left(f^{\prime}(r)\right)^{2}=0$ for every $r \in R$. Therefore $\left(f^{\prime}\right)^{2} \in N_{R}$.

$(1) \Rightarrow(3)$ By induction on $k$.

(3) $\Rightarrow(1)$ Let $k=2$. Then $F^{2}$ is induced by $x+2 f(x)$, and so that

$$
F^{2}(r+s \alpha)=r+s \alpha+2 f^{\prime}(r) s \alpha .
$$

While, by successive calculations,

$$
F^{2}(r+s \alpha)=F \circ F(r+s \alpha)=F\left(r+s \alpha+s f^{\prime}(r) \alpha\right)=r+s \alpha+s\left(2 f^{\prime}(r)+\left(f^{\prime}(r)\right)^{2}\right) \alpha .
$$

Then from the two expression of $F^{2}(r+s \alpha)$ follows that $s\left(f^{\prime}(r)\right)^{2}=0$ for every $r, s \in R$. Thus $\left(f^{\prime}(r)\right)^{2}=0$ for every $r \in R$, and hence $\left(f^{\prime}\right)^{2} \in N_{R}$.

In the following proposition we show that how FDP is useful in describing the behavior of the elements of the stabilizer group $S t_{\alpha}(R)$ in connection with their polynomial expressions.

Proposition 3.8. Let $F \in S t_{\alpha}(R)$. Suppose that $x+f(x)$ induces $F$, where $f \in N_{R}$. If $N_{R}$ satisfies FDP, then the following statements hold:

(1) $x-f(x)$ induces $F^{-1}$;

(2) $F^{k}=\underbrace{F \circ F \circ \cdots \circ F}_{k \text { times }}$ is induced by $x+k f(x)$ for every $k \in \mathbb{N}$;

(3) if $G \in S t_{\alpha}(R)$ is induced by $x+g(x)$, where $g \in N_{R}$, then $x+f(x)+g(x)$ induces $F \circ G$. 
Proof. Since $N_{R}$ satisfies FDP, we have $\left(f^{\prime}\right)^{2} \in N_{R}$, and hence (1) and (2) hold by Lemma 3.7 .

(3) Let $G \in S t_{\alpha}(R)$ be induced by $x+g(x)$, where $g \in N_{R}$. Then by FDP, $f^{\prime} g^{\prime} \in N_{R}$. Now we have for every $r, s \in R$, by Fact 3.1 and since $f, g \in N_{R}$,

$$
\begin{aligned}
G \circ F(r+s \alpha) & =G\left(r+s \alpha+s f^{\prime}(r) \alpha\right) \\
& =\left(r+s \alpha+s f^{\prime}(r) \alpha\right)+g\left(r+s \alpha+s f^{\prime}(r) \alpha\right) \\
& =\left(r+s \alpha+s f^{\prime}(r) \alpha\right)+\left(s+s f^{\prime}(r)\right) g^{\prime}(r) \alpha \\
& =r+s \alpha+s f^{\prime}(r) \alpha+s g^{\prime}(r) \alpha \text { by FDP. }
\end{aligned}
$$

Therefore $G \circ F$ is induced by the polynomial $x+f(x)+g(x)$.

We prove now a special case of [1, Theorem 4.1].

Lemma 3.9. Let $g \in R[x]$. Then $g$ is a permutation polynomial on $R[\alpha]$ if and only if $g$ is a permutation polynomial on $R$ and $g^{\prime}(r)$ is a unit for every $r \in R$.

Proof. $(\Rightarrow)$ Let $c \in R$. Then $c \in R[\alpha]$. Since $g$ is a permutation polynomial over $R[\alpha]$, there exist $a, b \in R$ such that $g(a+b \alpha)=c$. Thus $g(a)+b g^{\prime}(a) \alpha=c$ by Fact 3.1. So $g(a)=c$, whence $g$ is surjective on the ring $R$. Hence $g$ is a permutation polynomial on $R$.

Let $a \in R$ and suppose that $g^{\prime}(a)$ is a non-unit in $R$. Then $g^{\prime}(a)$ is a zero divisor of $R$. Let $b \in R, b \neq 0$, such that $b g^{\prime}(a)=0$. Then $g(a+b \alpha)=g(a)+b g^{\prime}(a) \alpha=g(a)$, so $g$ is not injective, which contradicts to the fact being bijective over $R[\alpha]$.

$(\Leftarrow)$ We need only to prove that $g$ is injective. For this let $a, b, c, d \in R$ such that $g(a+b \alpha)=g(c+d \alpha)$. Then $g(a)+b g^{\prime}(a) \alpha=g(c)+d g^{\prime}(c) \alpha$ by Fact 3.1. Thus we have $g(a)=g(c)$ and $b g^{\prime}(a)=d g^{\prime}(c)$. Hence $a=c$ since $g$ is a permutation polynomial on $R$. So, since $g^{\prime}(a)$ is a unit in $R, b=d$ follows.

We recall the following well-known result.

Lemma 3.10. [3, Theorem 3] Let $R$ be a local ring with nonzero maximal ideal $\mathfrak{m}$, and $g \in R[x]$. Then $g$ is a permutation polynomial on $R$ if and only if the following conditions hold:

(1) $g$ is a permutation polynomial on $R / \mathfrak{m}$;

(2) $g^{\prime}(r) \not \equiv 0 \bmod \mathfrak{m}$, for all $r \in R$.

Lemma 3.11. Let $R$ be a local ring with nonzero maximal ideal $\mathfrak{m}$, and $g \in R[x]$. Then $g$ is a permutation polynomial on $R[\alpha]$ if and only if $g$ is a permutation polynomial on $R$. 
Proof. $(\Rightarrow)$ Follows by Lemma 3.9.

$(\Leftarrow)$ Suppose that $g$ is a permutation polynomial on $R$. Then for all $a \in R$, $g^{\prime}(a) \not \equiv 0 \quad \bmod \mathfrak{m}$ by Lemma 3.10. Thus for all $a \in R, g^{\prime}(a)$ is a unit in $R$ since $R$ is a local ring. Hence $g$ is a permutation polynomial on $R[\alpha]$ by Lemma 3.9.

Corollary 3.12. Let $R$ be a local ring with nonzero maximal ideal $\mathfrak{m}$ and let $f \in$ $N_{R}$. If $F$ is the function induced by $x+f(x)$, then $F \in S t_{\alpha}(R)$.

In the rest of the paper let $N_{R}^{\prime}=\left\{f \in N_{R}: f^{\prime} \in N_{R}\right\}$. It is evident that $N_{R}^{\prime}$ is an ideal of $R[x]$ contained in $N_{R}$.

Lemma 3.13. Let $g \in R[x]$. Then $g$ is a null polynomial on $R[\alpha]$ if and only if $g \in N_{R}^{\prime}$.

Proof. By Fact 3.1, $g(a+b \alpha)=g(a)+b g^{\prime}(a) \alpha$ for every $a, b \in R$.

$(\Leftarrow)$ Immediately.

$(\Rightarrow)$ Since $g$ is null on $R[\alpha]$ we have that $g(a)+b g^{\prime}(a) \alpha=0$ for every $a, b \in R$. This is equivalent to $g(a)=b g^{\prime}(a)=0$ for every $a, b \in R$. Thus if $b=1$, we have $g(a)=g^{\prime}(a)=0$ for very $a \in R$. Hence $g \in N_{R}^{\prime}$.

We are now ready to prove our main result for this section.

Proposition 3.14. Let $R$ be a local ring with nonzero maximal ideal $\mathfrak{m}$. If $N_{R}$ satisfies FDP, then

$$
S t_{\alpha}(R) \cong N_{R} / N_{R}^{\prime} .
$$

Proof. Let $f \in N_{R}$, then obviously $[x+f(x)] \in S t_{\alpha}(R)$ by Corollary 3.12 , where $[x+f(x)]$ denotes the function induced by $x+f(x)$ on $R[\alpha]$.

Now define a function $\psi: N_{R} \longrightarrow S t_{\alpha}(R)$ by $\psi(f)=[x+f(x)]$. By Proposition 3.6, $\psi$ is surjective. Let $g \in N_{R}$. Then set $F_{1}=[x+f(x)], F_{2}=[x+g(x)]$ and $F_{3}=[x+f(x)+g(x)]$. By Proposition 3.8, $F_{1} \circ F_{2}=F_{3}$. Therefore $\psi(f+g)=\psi(f) \circ \psi(g)$, whence $\psi$ is a homomorphism. Hence $N_{R} / \operatorname{ker} \psi \cong S t_{\alpha}(R)$ by the first isomorphism theorem.

Now, $\operatorname{ker} \psi=\left\{f \in N_{R}:[x+f(x)]\right.$ is the identity permutation on $\left.R[\alpha]\right\}$. By Lemma 3.13, $N_{R}^{\prime} \subseteq \operatorname{ker} \psi$. On the other, if $f \in \operatorname{ker} \psi$, then $x+f(x)$ induces the identity on $R[\alpha]$. Hence $x+f(x)=x+h(x)$ for some null polynomial (on $R[\alpha]$ ) $h \in R[\alpha][x]$ by Lemma 3.4. Thus $f=h$ and $f$ is a null polynomial on $R[\alpha]$. Since $f \in R[x]$ we have $f \in N_{R}^{\prime}$ by Lemma 3.13. Therefore ker $\psi \subseteq N_{R}^{\prime}$.

Remark 3.15. In [1], for the case $R=\mathbb{Z}_{p^{n}}$ the ring of integers modulo $p^{n}$, it was only proved that $\left|S t_{\alpha}\left(\mathbb{Z}_{p^{n}}\right)\right|=\left[N_{\mathbb{Z}_{p^{n}}}: N_{\mathbb{Z}_{p^{n}}}^{\prime}\right]$, for every $n>1$, and it was unclear 
whether $S t_{\alpha}\left(\mathbb{Z}_{p^{n}}\right)$ and $N_{\mathbb{Z}_{p^{n}}} / N_{\mathbb{Z}_{p^{n}}}^{\prime}$ are isomorphic or not. But, now Proposition 3.14 tells us they are isomorphic via a map induced by the function $\psi$ defined in the above proof, when $N_{R}$ satisfies FDP.

Corollary 3.16. Let $R$ be a local ring with nonzero maximal ideal $\mathfrak{m}$. The function $\psi: N_{R} \longrightarrow S t_{\alpha}(R)$, defined by $\psi(f)=[x+f(x)]$ for every $f \in N_{R}$, is a homomorphism if and only if $N_{R}$ satisfies FDP.

Proof. $(\Leftarrow)$ Follows by the same argument given in the proof of the previous proposition.

$(\Rightarrow)$ Assume that $\psi$ is a homomorphism. Let $f, g \in N_{R}$. Put $F_{1}=[x+f(x)], F_{2}=$ $[x+g(x)]$ and $F_{3}=[x+f(x)+g(x)]$. Then $F_{1}, F_{2}, F_{3} \in S t_{\alpha}(R)$ by Corollary 3.12 . We now consider $\psi(f+g)=[x+f(x)+g(x)]=F_{3}$. But, since $\psi$ is a homomorphism by assumption, we have that $\psi(f+g)=\psi(f) \circ \psi(g)=F_{1} \circ F_{2}$. Thus $F_{1} \circ F_{2}=F_{3}$. Now, for every $a, b \in R$, we have

$$
F_{1} \circ F_{2}(a+b \alpha)=a+b \alpha+b\left(g^{\prime}(a)+f^{\prime}(a)+f^{\prime}(a) g^{\prime}(a)\right) \alpha,
$$

and $F_{3}(a+b \alpha)=a+b \alpha+b\left(f^{\prime}(a)+g^{\prime}(a)\right) \alpha$.

Hence $b f^{\prime}(a) g^{\prime}(a) \alpha=0$ for every $a, b \in R$, which implies that $f^{\prime}(a) g^{\prime}(a)=0$ for every $a \in R$. Thus $f^{\prime} g^{\prime} \in N_{R}$, and so $N_{R}$ satisfies FDP.

Remark 3.17. The function $\psi$ defined in Corollary 3.16 seems natural in the sense that it sends every polynomial $g \in N_{R}$ to the function induced by $x+g(x)$ over $R[\alpha]$, however, we notice the following.

(1) When $R=\mathbb{F}_{q}$, the function $\psi$ is not defined. For instance, take $f(x)=$ $x^{q}-x \in N_{\mathbb{F}_{q}}$, but $F=[f(x)+x]=\left[x^{q}\right] \notin S t_{\alpha}\left(\mathbb{F}_{q}\right)$ since $F$ is not a permutation as $F(0)=F(\alpha)=0$ (compare this with Remark 2.13-(1)).

(2) If $R=\mathbb{Z}_{8}$, the function $\psi$ can be defined by Corollary 3.12. But, by Remark 2.13-(2), $N_{\mathbb{Z}_{8}}$ does not satisfy FDP. So $\psi$ is not a homomorphism by Corollary 3.16 .

Applying Proposition 3.14 to Corollary 2.14 gives the following result.

Corollary 3.18. Let $p$ be a prime number and $n$ a positive integer.

(1) If $p>2$, then $S t_{\alpha}\left(\mathbb{Z}_{p^{n}}\right) \cong N_{\mathbb{Z}_{p^{n}}} / N_{\mathbb{Z}_{p^{n}}}^{\prime}$ for every $1<n \leq p+1$.

(2) If $p=2$, then $S t_{\alpha}\left(\mathbb{Z}_{4}\right) \cong N_{\mathbb{Z}_{4}} / N_{\mathbb{Z}_{4}}^{\prime}$.

We conclude the paper by showing that the null ideal on dual numbers satisfies FDP. For this we recall the following fact from [1]. 
Lemma 3.19. [1, Theorem 3.5] Let $R$ be a commutative ring and let $A=R[\alpha]$ be the ring of dual numbers over $R$. Let $f=f_{1}+f_{2} \alpha$, where $f_{1}, f_{2} \in R[x]$. Then $f \in N_{A}$ if and only if $f_{1} \in N_{R}^{\prime}$ and $f_{2} \in N_{R}$.

Proposition 3.20. Let $R$ be a commutative ring and let $A=R[\alpha]$ be the ring of dual numbers over $R$. Then $N_{A}$ satisfies FDP.

Proof. Let $f, g \in N_{A}$. Then $f=f_{1}+f_{2} \alpha$ and $g=g_{1}+g_{2} \alpha$ for some $f_{1}, f_{2}, g_{1}, g_{2} \in$ $R[x]$ such that $f_{1}, g_{1} \in N_{R}^{\prime}$ and $f_{2}, g_{2} \in N_{R}$ by Fact 3.2 and Lemma 3.19 , respectively. But then $f_{1}^{\prime} g_{1}^{\prime} \in N_{R}^{\prime}$ and $f_{1}^{\prime} g_{2}^{\prime}+f_{2}^{\prime} g_{1}^{\prime} \in N_{R}$. Thus, by Lemma 3.19,

$$
f^{\prime} g^{\prime}=f_{1}^{\prime} g_{1}^{\prime}+\left(f_{1}^{\prime} g_{2}^{\prime}+f_{2}^{\prime} g_{1}^{\prime}\right) \alpha \in N_{A} .
$$

Acknowledgement. I would like to thank the reviewers for their suggestions and helpful comments. I am also very grateful to Chimere Anabanti for his useful comments on an earlier version of the manuscript.

\section{References}

[1] H. Al-Ezeh, A. A. Al-Maktry and S. Frisch, Polynomial functions on rings of dual numbers over residue class rings of the integers, Math. Slovaca, 71(5) (2021), 1063-1088.

[2] B. R. McDonald, Finite rings with identity, Pure and Applied Mathematics, Vol. 28, Marcel Dekker, Inc., New York, 1974.

[3] A. A. Necaev, Polynomial transformations of finite commutative local rings of principal ideals, Math. Notes, 27(5-6) (1980), 425-432, translate from Mat. Zametki, 27(6) (1980), 885-897.

[4] W. Nöbauer, Über die Ableitungen der Vollideale, Math. Z., 75 (1961), 14-21.

[5] M. W. Rogers and C. Wickham, Polynomials inducing the zero function on local rings, Int. Electron. J. Algebra, 22 (2017), 170-186.

\section{Amr Ali Al-Maktry}

Institute of Analysis and Number Theory (5010)

Technische Universität Graz

kopernikusgasse 24/II

8010 Graz, Austria

e-mail: almaktry@math.tugraz.at 